\title{
SURFACE CONSOLIDATION OF WALL PAINTINGS USING LIME NANO-SUSPENSIONS
}

\author{
JAN VOJTĚCHOVSKÝ
}

\begin{abstract}
Studio of Restoration and Conservation of Wall painting and Sgraffito, Faculty of Restoration, University of Pardubice, Jiráskova 3, Czech Republic

correspondence: Jan.Vojtechovsky@upce.cz
\end{abstract}

\begin{abstract}
Within the field of the conservation of historical and cultural monuments, lime nanosuspensions are still a relatively new and unexplored material. This study examines their effect on the consolidation of architectural surfaces and, consequently, on wall paintings. Previous experiments showed that considerably deteriorated materials may not be adequately strengthened using only lime nano-suspensions. Therefore, the effects of their admixtures and gradual applications with silicic acid esters were examined. For verification, a simulation of a deteriorated lime-based paint layer was created on panels of plaster. The results of the consolidation were subsequently studied using objective (peeling test, water absorption capacity test, measuring colour changes using a mobile spectrophotometer) and subjective methods (comparison of visual changes to a set standard and by testing cohesion using a cotton swab). The microstructure of a consolidated paint layer was studied with a scanning electron microscopy. Tests proved that with either individual lime-alcoholic suspensions or with successive applications and mixtures of silicic acid esters it is feasible to achieve good consolidation results, whilst the alkoxysilane content of the agent indisputably increases the consolidating effect of these materials.
\end{abstract}

KEYWORDS: lime nano-suspensions; silicic acid esters; surface consolidation; conservation; wall painting.

\section{INTRODUCTION}

In recent years, the consolidation of paint layers of wall paintings or architectural surfaces is, as in other areas of the care of monuments, associated with the notion of material compatibility. On the basis of this notion, materials using the same type of binder initially used in the original artefact are being increasingly applied in the consolidation and conservation of historical and culturally significant buildings and artefacts. A result of the above-mentioned efforts is that a group of consolidating agents was developed at the beginning of the new millennium - so-called "lime nano-suspensions" [1. As they are materials whose only consolidation agent is the calcium hydroxide $\mathrm{Ca}(\mathrm{OH})_{2}$, they are designated for strengthening other porous calcareous materials such as lime plasters and their surface layers (washes, paintings). As opposed to a traditional lime-based consolidant, which is a saturated solution of the $\mathrm{Ca}(\mathrm{OH})_{2}$ in water (limewater), these consolidants have a higher concentration of the active component and, moreover, the consolidant is dispersed in an alcoholic media, which, in many cases, as in the case of risks connected with moisture, can be more advantageous (for instance, in case of activation of water soluble salts). The aim of this study was to investigate the effects and risks of strengthening lime based paintings and surface layers of historical plasters using this type of consolidant as well as its modifications.

In spite of the fact that, as opposed to limewater, the concentration of the active matter in lime-alcoholic suspensions is higher, in the cases where the strength- ened substrate had disintegrated excessively, the consolidating effect was still inadequate. Therefore, the tests included modifications of these suspensions with silica based consolidants, where the resulting content of the solid component is approx. 10 times higher. On the basis of previous experiments described in literary sources [2], the decision was made to test mixtures and combinations of silicic acid esters, which, in the conservation field, are the most commonly used consolidants for porous inorganic materials, including plaster, renderings and their surfaces.

\section{BASIC CHARACTERISTICS OF LIME ALCOHOLIC SUSPENSIONS}

The term lime-alcoholic nano-suspensions (or nanosols) is used for suspensions of calcium hydroxide in aliphatic alcohols with particles of calcium hydroxide, which size is about 50 to $300 \mathrm{~nm}$. Theoretically, they are not nanomaterials in the true sense as their size would have to be $100 \mathrm{~nm}$ at the most 3 .

The individual suspensions, available commercially or as an experimental developmental material, differ from each other in their particle morphology, their concentration or type of alcohol. The most common dispersing agents are ethanol, 1-propanol and 2-propanol.

The suspensions are produced in various concentrations, commercially available in concentrations from 5 to $50 \mathrm{~g}$ of $\mathrm{Ca}(\mathrm{OH})_{2}$ to 11 alcohol. The viscosity and "whiteness" of the suspensions differ partly according to their concentration. Suspensions with higher concentrations have slightly higher viscosity and they 
are more opaque, "whiter". So far, two manufacturers offer products commercially - products from the German manufacturer IBZ Salzchemie, marketed under the CaLoSil® brand [4, and those from the Italian manufacturer CSGI [5], marketed under the Nanorestore Plus ${ }^{\circledR}$ brand (formerly Nanorestore ${ }^{\circledR}$ ). Other similar materials are still under development or in their experimental phase. Compared to other dispersions (e.g., polymer dispersions) the stability of lime-alcoholic nanosuspensions is lower (only ca. 3 months), probably due to the small size of the particles which tend to agglomerate much faster. One manufacturer guarantees stability of its product in an unopened packaging for 12 months 6 .

According to the needs, the suspension can be diluted by adding organic solvents. Usually, the same alcohol in which the $\mathrm{Ca}(\mathrm{OH})_{2}$ particles have been dispersed. However, other options for diluting have been published. One of these can be to dilute with a mixture of ethanol and acetone to a ratio of $40: 60$, which, according to the authors of this article, should ensure better distribution of the consolidant in the material to be strengthened [7].

After application of the suspension to the material to be strengthened, the dispersion medium (alcohol) evaporates completely. This can result in the re-migration of $\mathrm{Ca}(\mathrm{OH})_{2}$ particles toward the surface, which itself can cause white haze on the material being strengthened. Restricting this process can be carried out in several ways, by subsequent wetting with organic solvents or water (something dealt elsewhere within this study), by the aforementioned dilution of the suspension with a mixture of ethanol and acetone or by applying solutions of cellulose derivatives in a low concentration to the surface of the treated object after the consolidation [8]. Another reason for the creation of the white haze can be an accumulation of the consolidant on the surface during its application. According to in situ tests, it seems that a limiting factor could be not only the pore size of the strengthened material (including thin surface layers), but possibly also a thin layer of deposits or secondary interventions adhering to the surface.

\section{EXPERIMENT DESCRIPTION}

The main aim of the experiment was to study the effect of individual lime-alcoholic suspensions, which were examined at the Faculty of Restoration, University of Pardubice within the framework of two international research projects, STONECORE [9] and NANOFORART [10]. The experiment was partly carried out during the course of the second of the abovementioned projects in whose framework some of its results were presented.

Another aim, deemed necessary during the course of the experiment, was the testing of mixtures and combinations of lime-alcoholic suspensions with silicic acid esters. This requirement arose primarily as a result of a restoration work on the wall paintings in the dome of
St. Isidor Chapel in Křenov near Moravská Třebová, where the question of consolidating the severely deteriorated lime-based paint layers was an issue. Organic based consolidants as synthetic or natural polymers were resolutely rejected here due to the persistent high relative humidity in the building and possible risks related to the changed behaviour of paint layer treated by these consolidants (e.g., dilatation, water vapour permeability, biodegradation). Based on previous experiences [11, the necessary level of consolidation was impossible to achieve using (ideally compatible) lime-alcoholic suspensions.

In order to carry out comparison tests of the consolidants, five lime-alcoholic suspensions tested within the framework of the NANOFORART project were chosen (two of which are commercially available today under the brand names Nanorestore Plus ${ }^{\circledR}$ Ethanol 10 (CSGI) and Nanorestore Plus® Propanol 10 (CSGI). The product CaLoSil® E25 (IBZ Salzchemie), tested within the STONECORE project, was selected for comparison.

For the planned creation of more effective consolidants, silicic acid esters (oligomeric alkoxysilanes) in different forms were included in the experiment. Unmodified alkoxysilane (KSE 100 and KSE 300), its elasticized modification (KSE $300 E$ ) and a product modified with special primers for improving adhesion to the materials bound with calcium carbonate (KSE $300 \mathrm{HV}$ ) were used. Products from the manufacturer Remmers were chosen precisely because they offer agents in all our required categories whose examination was planned [12]. During the experiment, not only the application of the individual consolidants (above all lime-nanosuspensions) was carried out, but also the application of their mixtures and subsequent application to test panels with the focus on the effect of the combined application of both abovementioned groups of materials was tested.

\subsection{Characteristics OF THE TEST SUBSTRATES}

On the basis of previous experience, it was decided that testing the effects of all selected consolidants and their combinations or mixtures would be carried out on plaster panels with a simulation of a deteriorated paint layer. Due to the heavily damaged paint layers and plaster of the ceiling paintings in St. Isidor Chapel in Křenov, where the resulting technology was to be eventually applied, it was decided to simulate not only the completely powdered paint layer, but also the weakened plaster layering. On that basis, two plaster panels were prepared with a model simulation of the plaster and paint layer deterioration. Panels were prepared ca. 2 month before the application of the consolidants started. Nevertheless, a test of maturity of the substrate (complete conversion of $\mathrm{Ca}(\mathrm{OH})_{2}$ to $\mathrm{CaCO}_{3}$ ) wasn't realized, because the simulation of the paint layer contained no binder and the experiment did 


\begin{tabular}{lll}
\hline \multicolumn{1}{c}{ Arricio } & \multicolumn{1}{c}{ Intonaco } & \multicolumn{1}{c}{ Paint layer } \\
\hline - 1 part lime putty & $\bullet$ 1 part lime putty & $\bullet$ parts burnt umber reddish \\
- 4 parts sand from Kostelecké & $\bullet$ 2 parts limestone powder & pigment (Kremer Pigmente - \\
Horky sand-pit sieved & $\bullet 5$ parts sand from Kostelecké & product No. 40700) \\
to $d<2 \mathrm{~mm}$ & Horky sand-pit sieved & $\bullet$ 1 part limestone powder \\
& to $d<0.5 \mathrm{~mm}$ & $\bullet$ 10 parts distilled water \\
\hline
\end{tabular}

TABle 1. Composition of individual plaster panel layers (parts by volume).

not focus on the interactions between the consolidant and the plaster.

Two layers of a lime plaster with a low binder content were applied to the test panels. Whilst the first layer of the rough plaster (arricico) was scraped when still moist and not hardened, the finer plaster layer (intonaco) was applied and compacted with a trowel and subsequently levelled with a wooden float covered in felt. Two layers of paint without a binder were brushed onto the intonaco layer. In order to simulate deteriorated paintings using fresco 13 or lime secco [14 techniques as closely as possible, a limestone powder (calcium carbonate) was added to the pigment. This commonly functions as a binder, filler or pigment in the paint layer. For the composition of the individual layers in parts by volume see Table 1 .

\subsection{Characteristics OF APPlied Methods}

During the experiment, the main intention was to study the resulting consolidation rate and apparent as well as possible hidden negative impacts on the treated material. For evaluation of the resulting strengthening effect on paint layers, there are not many objective methods. Probably, the peeling test, where an adhesive tape is used to determine the strengthening gain, could be considered as the most widespread method. The principle of the method is based on determining the mass of the peeled tape with defined dimensions before and after the consolidation. Even though this method has several more or less objective varieties, in our experiment, it does not provide correctly interpretable results for evaluating the strengthening effect on the used simulation of the damaged paint layer. With an increase of paint layer cohesion due to the consolidation, the amount of the material peeled off can also increase. This can lead to an incorrect evaluation of the results. In order to eliminate the inaccuracies of this test, it was decided to carry out the test in two different forms, with different adhesion power of the tape used.

For the same reason, another objective method in the form of a sclerometric hardness test was intended. However, as the painted layer of a wall painting, especially unconsolidated, is an extremely sensitive material, the most delicate method of this type of testing was required. The so-called Wolff-Wilborn test, which uses the varying hardness of graphite pencils to evaluate the hardness of materials, seemed to meet this requirement. During a previous test [15], it was evi- dent that the evaluation of the test was inconclusive and thus highly subjective. Therefore, the decision was made to eliminate this method from the following experiment.

On the basis of previous experience in the conservation-restoration field, it was evident that even subjective methods could provide credible and helpful results. Subjective evaluation methods (for example visual observations), as a helpful tool for assessment in restoration and conservation field, are mentioned in many publications [16, 17]. In addition, restorers are used to working with and subsequently evaluate these kinds of methods. A swab test of the paint layer with the help of a cotton wool tampon followed by a visual evaluation of the amount of material removed was the method chosen to complement objective methods of evaluating the strengthening rate. Visual changes (hazing or darkening) were also subjectively evaluated. However, visual changes were also evaluated objectively with the help of a Konica Minolta CM2600d mobile spectrophotometer, which enables the recording of a colour space using the CIELAB [18] system.

The structure of samples taken from the surface were studied using Scanning Electron Microscopy $(S E M)$ to evaluate less evident, direct and indirect effects of consolidation and the micromorphology of the substrate as well as the newly added binder. This was carried out using a Mira 3 LMU (Tescan) electron microscope with EDS analysis Quantax 2000 (Bruker). The measurement was undertaken on fragments of simulated damaged paint layers in a high-vacuum mode using Secondary Electron $(S E)$ and Back-Scattered Electron Detectors $(B S E)$. Samples were sputtered with gold. The aim of this microscopic study was to ascertain the distribution of the consolidant within the pore system of the strengthened substrate, the microstructure of the resulting binder and the eventual changes of the pore system after the consolidation.

In order to evaluate long-term influences affecting the tested substrate, it is usually important whether or not the surface closes up or whether border lines form between the strengthened and un-strengthened zones. In this case, there are not many possibilities for evaluating the consolidation of paint layers. Probably the most common one is evaluating changes of water absorption. A method was sought, which would be adequately sensitive, would provide the relevant results and, at the same time, be available and easily 


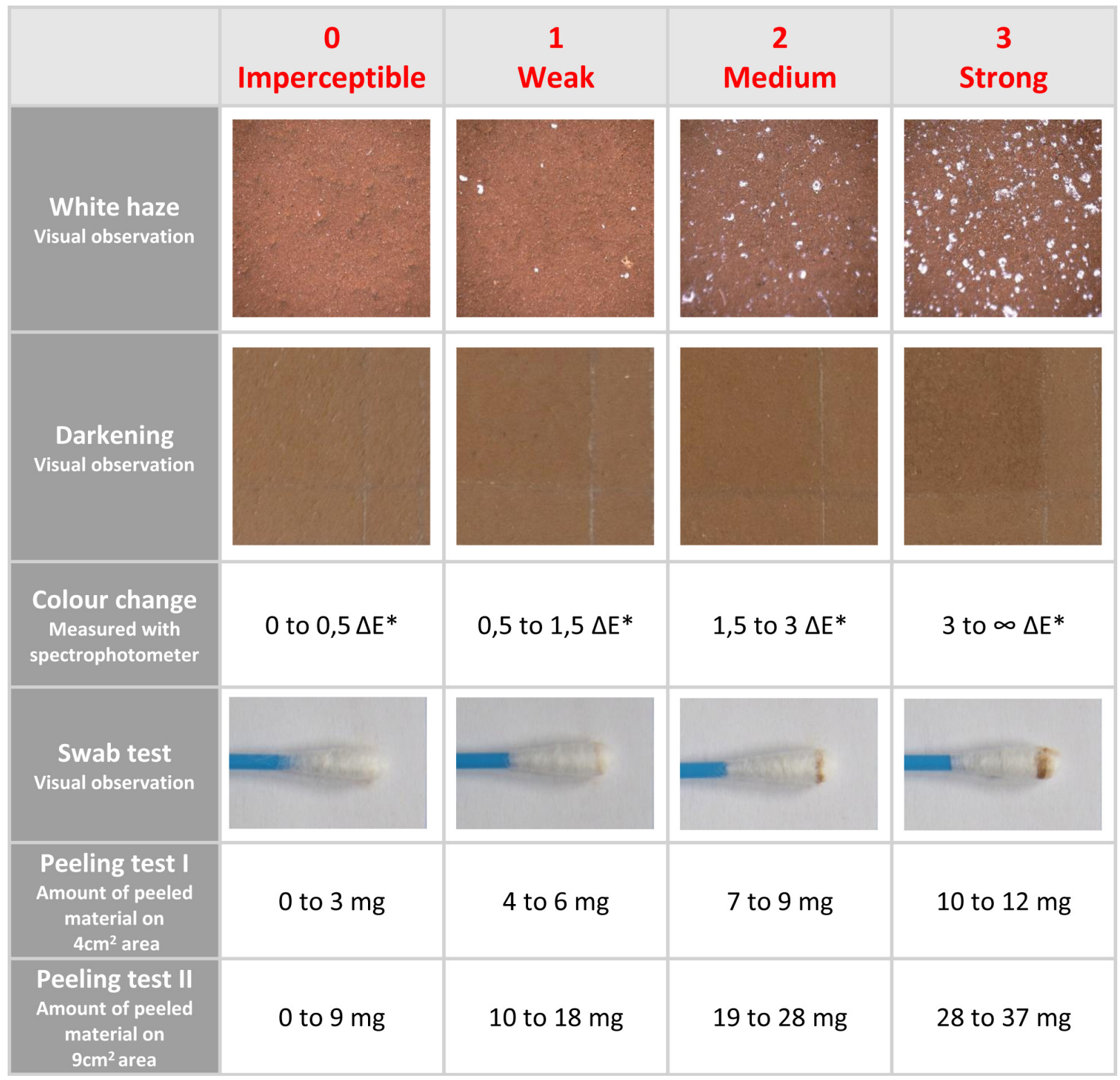

TABLE 2. Table showing methods of assessing the consolidation and related unwanted effects, defined after the application of consolidating agents on the basis of threshold values

applicable (especially due to its intended use in situ). A traditional evaluation of water absorption using the Karsten tube method in the case of delicate paint layers was inapplicable. An alternative could have been the Mirowski Pipe or its modified or automated form [19]. In the end, the decision was made to use the Contact Sponge Method [20], which is extremely simple, undemanding and could be used for measuring directly on the artefacts on which the lime nanosuspensions and their mixtures with alkoxysilanes had been applied. This method was lightly-modified for the purposes of this experiment. The original sponge was replaced with a micro-porous polyvinyl alcohol sponge with the dimensions $30 \times 33 \times 74 \mathrm{~mm}$ (corresponding to the original dimensions $174 \times 33 \times 74 \mathrm{~mm})$. Measuring was carried out by applying the sponge to the surface of the panel through a Japanese tissue. The panel was placed in a horizontal position, so there was probably an influence of the gravity force as well. The sponge was applied in 5 cycles each lasting $60 \mathrm{~s}$ - always with $35 \mathrm{~s}$ intervals. At the beginning of the experiment, the sponge was weighed in order to study the absorption rate over time.

A comparatively important feature of lime nanosuspensions to measure is their stability, which, as it was already mentioned, in comparison with other commonly used consolidants is markedly lower. Stability was evaluated on the basis of the sedimentation rate of individual studied suspensions. The stability of mixtures with alkoxysilanes was evaluated on the basis of the mixture gelation rate.

\subsection{System FOR EVAluAting RESUlts}

One of the tasks of the experiment was to compile a logical evaluation system, which would clarify the measured data and allow a more clear interpretation. 


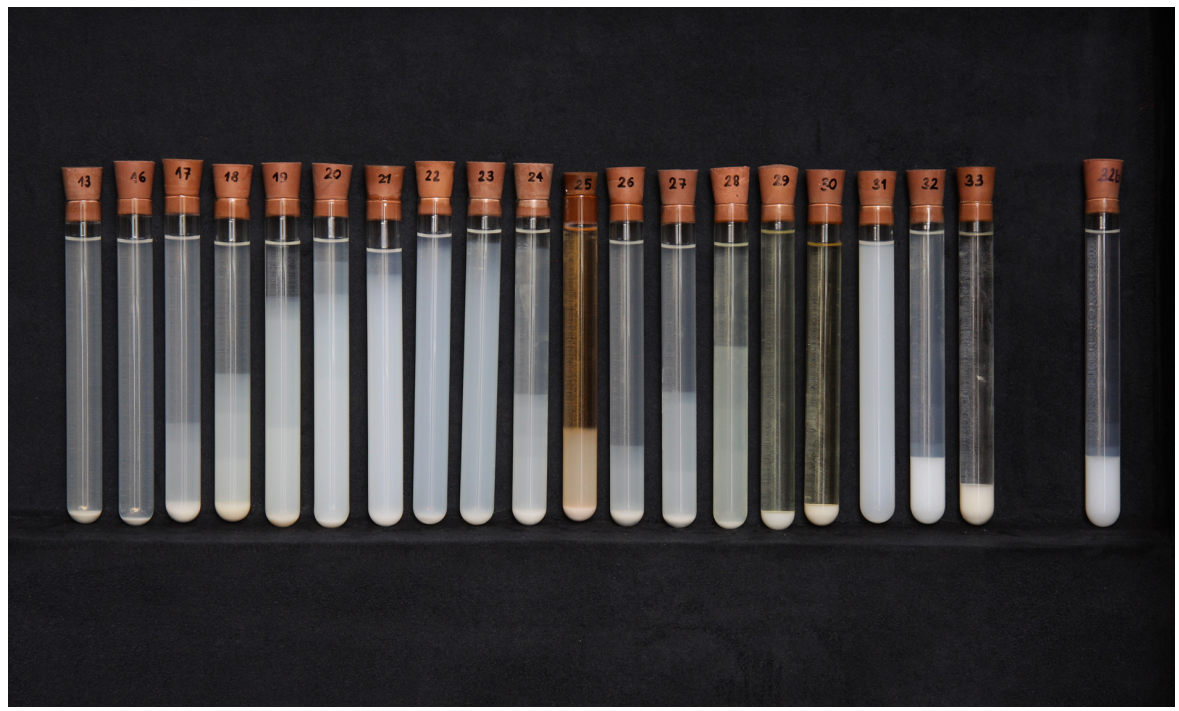

FiguRE 1. Sedimentation process of selected consolidants over 57 days. Test tubes 13,16 and 17 contain lime suspensions diluted to $5 \mathrm{~g} / \mathrm{l}$, test tubes 28-33 contain lime suspensions mixed with silicic acid esters.

In order to describe and enter the changes, which take place after the application of consolidants into one logical system, an attempt was made to evaluate these changes on a numbered scale from 0 to 3 . "0" marks the best result, meaning that there was a good consolidation or there were no significant visual changes after the application of the consolidant. Conversely, " 3 " marks the worst result. Therefore, the higher the number on the scale the worse the consolidation and the greater the unwanted effects on the consolidated area.

However, as the measurements and comparisons of methods showed, not all tests could be transcribed into values on a simple scale. Therefore, some tests, such as those dealing with the stability, water absorption and microstructure studies using Scanning Electron Microscopy, were evaluated individually. For the above described evaluation scale, see Table 2

In conclusion, alongside the evaluation of the individual tests, an overall mark has been calculated for each test field. This is an average mark given for visual impact and effectiveness of the consolidation.

\section{Results}

\subsection{Stability test of Selected CONSOLIDANTS}

All tested suspensions and some of their mixtures with alkoxysilanes were initially tested for stability. The suspensions and mixtures were placed into test tubes and photo-documentation was carried out at regular intervals.

The individual suspensions displayed vast differences in the stability. Due to the difficulties in accumulating all the tested consolidants, tests were carried out, on average, within 1 month of their production. Full stability (no sediment appearing at the bottom of the test tube) of the suspension ranged from between several days to ca. 8 weeks from the start of the experiment in the case of samples displaying the best results. This means that the full stability in the case of the most successful suspensions is reached in ca. 3 months. However, it is important to note that after a certain period, the sediment can be dispersed into the suspension again, as one manufacturer notes, after shaking the closed bottle or by an ultrasonic treatment 21]. The question remains as to whether the original properties of the suspension remain intact - above all the penetration ability.

Regarding stability of the mixtures of lime-alcoholic suspensions with alkoxysilanes, an acceptable level of stability was recorded in the case of mixtures with alkoxysilanes modified for a use on carbonate materials. In one case, this was determined to be 8 days. Nevertheless, for application purposes, stability is necessary only over a period of several hours for a safe application after mixing. For an example of results of the stability test, see Figure 1

\subsection{Tests CARRIED OUT ON TEST PANELS}

Two $100 \times 50 \mathrm{~cm}$ plaster testing panels were divided into a grid of $9 \times 9 \mathrm{~cm}$ sections. The consolidants were applied on the panels in a vertical position with a fine sprayer until the substrate was saturated when the suspension remained on the surface longer than several seconds. This process was derived from previous experiments. During the application, the values of relative humidity varied between $35-40 \%$. Immediately after the application, the panels were placed under an airtight foil maintained with relative humidity of around 55-65\%. The air temperature was recorded in the range of $20-22{ }^{\circ} \mathrm{C}$. The role of the relative humidity was already tested in the previous experiment [22] and it appeared to be significant. It showed that in the relative humidity around $30 \%$, a stronger white haze is formed than in the relative humidity around $60 \%$. The tests were carried out on 


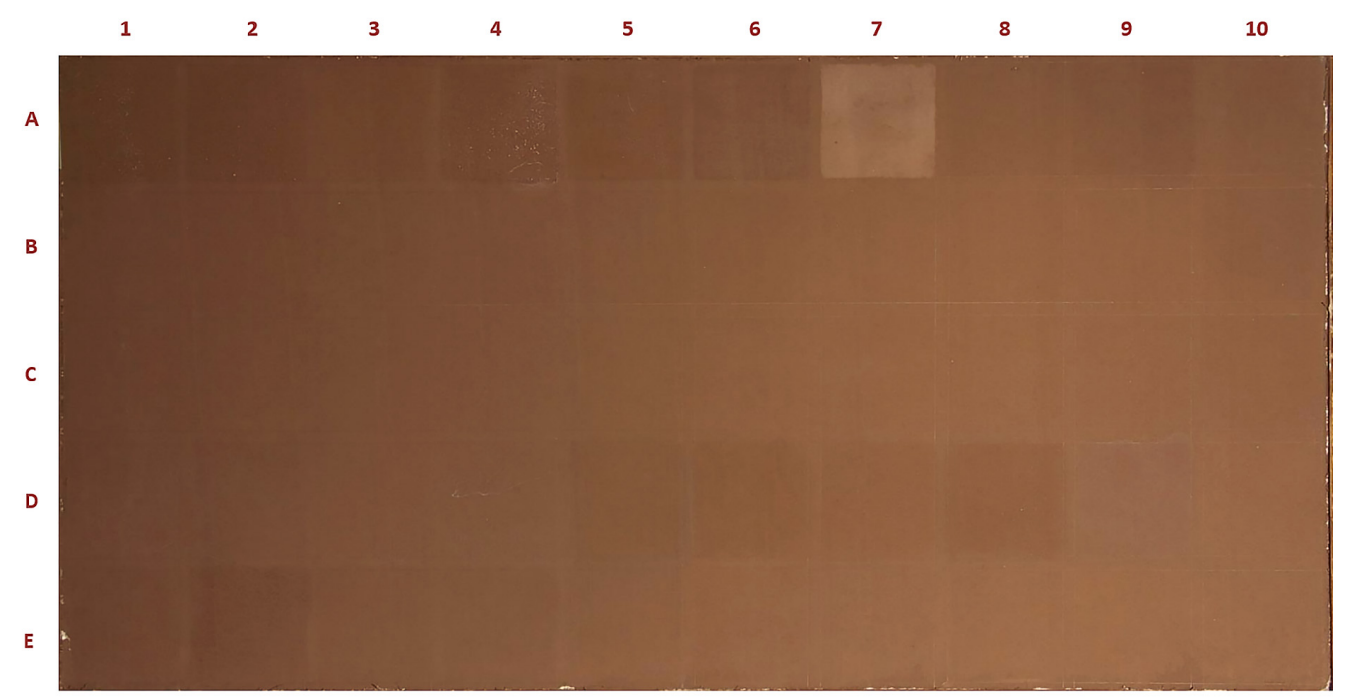

Figure 2. Condition after application of all consolidants on one of the test panels.

both lime suspensions diluted with various alcohols to a concentration of 5 and $10 \mathrm{~g}$ of $\mathrm{Ca}(\mathrm{OH})_{2}$ per 1 litre of agent and subsequent applications of silicic acid esters (alkoxysilanes) and lime suspensions as well as on their mixtures. Figure 2 shows one of the panels after testing.

Whilst the subjective evaluation tests (white haze, darkening, swab test) were realized after two weeks, part of the objective tests was realized after two months (spectrophotometer measuring, peeling tests I and II) and the other part after more than one year (water absorption test).

For the purpose of the evaluation, result sheets were created, which summarize the tests carried out on each test section. Each square is described on the sheet in detail, with the type of the consolidant and its concentration, followed by a detailed description concerning application - date, number of cycles, the amount of the consolidant used (volume) and details about eventual subsequent wetting. Last but not least, marks (scores) were given according to the results of individual tests. Six marks for each individual test were averaged out into a one overall mark, which serves as a mutual comparison of the test sections. Each sheet contains photo-documentation of each field after application of the consolidant, marking off of the test sections (peeling tests, swab test, water absorption test), an image taken using a USB microscope and a photograph documenting both peeling and swab tests. Figure 3 shows one of these sheets.

\subsection{Study AND Evaluation}

\section{OF MICROSTRUCTURE}

Studies of the microstructure of consolidated samples were carried out using Scanning Electron Microscopy as mentioned above. Representatives of the individual consolidating systems were chosen for the studies - the application of lime suspensions in several cycles, gradual application of alkoxysilane and lime suspension based agents and a mixture of both abovementioned consolidants. One test section was also included where alkoxysilane, its mixture with lime suspension and a pure lime suspension were subsequently applied. For the purpose of the comparison, a sample was also taken from a test section consolidated with a twocycle application of alkoxysilane consolidants.

The images of the microstructure (Figure 4 ) show that each of the aforementioned groups displays a different binder microstructure. Generally, it can be said that the binder accumulates on the walls of the pores and in the contact zones between the grains of the substrates thus forming new connections or bridges between these grains. Contrary to gels forming during the hardening of alkoxysilane consolidants, the binder structure formed through the combinations or mixtures (or both) is more porous or contains a greater number of shrinkage micro-cracks. In all three casesm the binding of the gel to the grains of the substrate is good and new binder does not show the tendency to detach from these grains. In the case of samples consolidated purely with lime suspensions, it was not possible to differentiate conclusively the new binder from the substrate itself. This is due firstly to the composition of the new binder, which is chemically identical to the particles present in the substrate (the paint layer contains $\mathrm{CaCO}_{3}$ as a filler), and also due to the small amount of the consolidant applied and probably also due to its microstructure which is highly porous. Generally, it could be said that the porosity of the substrate simulating a deteriorated paint layer is in no way affected by the consolidation. Therefore, it can be supposed that not even those physical properties related to the porosity, such as vapour permeability or water absorption capacity (WAC), would change significantly as a result of the consolidation. This was confirmed by the values of the water absorption coefficient measured. The measurement of the WAC was performed after more than one year 


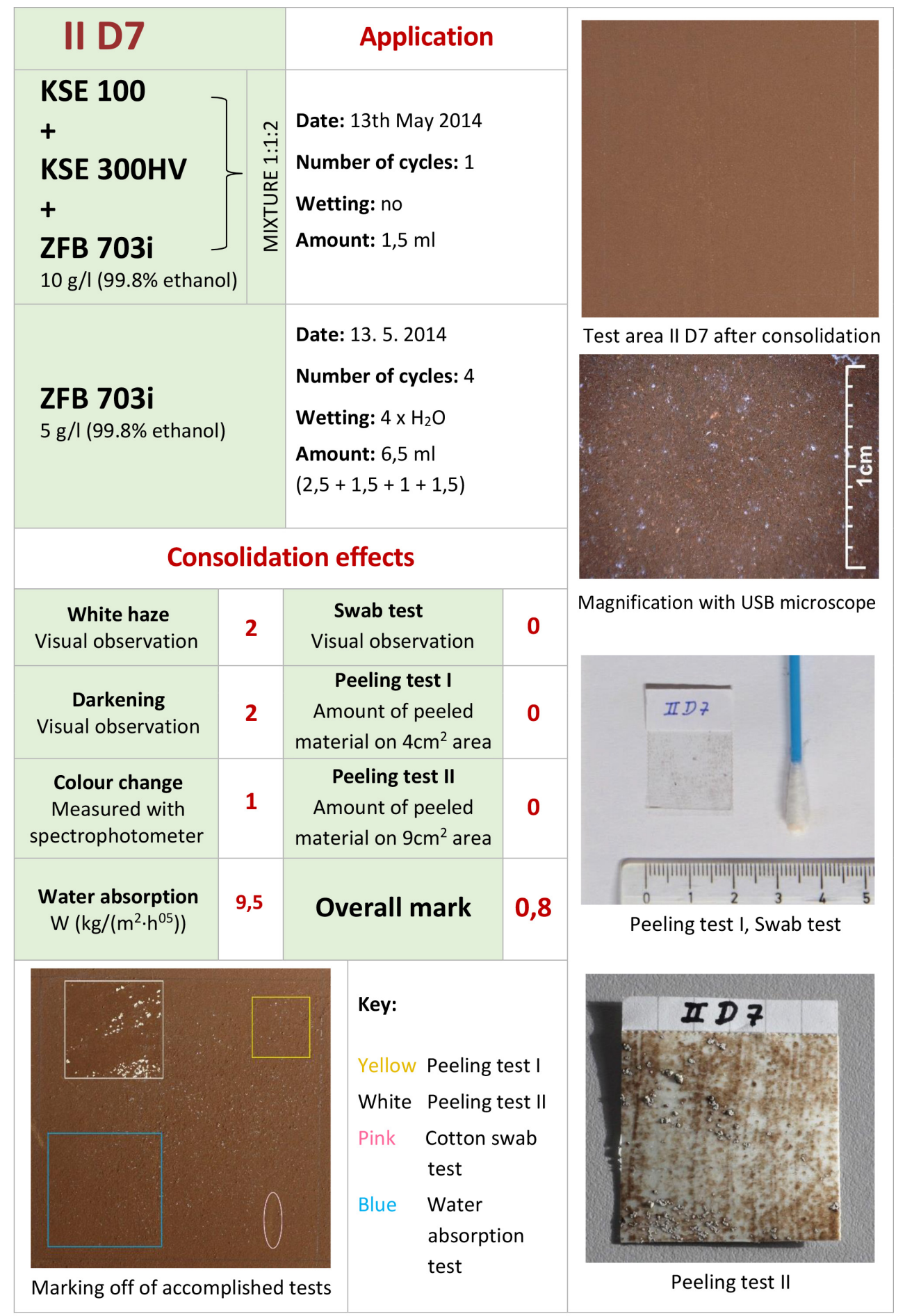

Figure 3. Test sheet for one of the test sections (IID7). It describes a successive application of a $1: 1: 2$ mixture and lime-alcoholic suspension in a concentration of $5 \mathrm{~g} / \mathrm{l}$.

after the consolidation, so the effect of the temporary hydrophobicity of alkoxysilanes can be considered as negligible.

\section{Discussing the Results}

Within the framework of the experiment, the individual suspensions were compared and various types of alcohol were tested for diluting them. On one hand, as already mentioned above, the full stability of the most stable suspensions is only approx. 3 months from its production. On the other hand, the suspension can be re-dispersed after shaking or an ultrasonic treatment, giving still acceptable consolidation results for a certain period. A fundamental finding was discovered during the study of the effects of the denatured [23] and $99.8 \%$ ethanol 24. Denatured ethanol, which should contain more water, exhibited very good ef- 

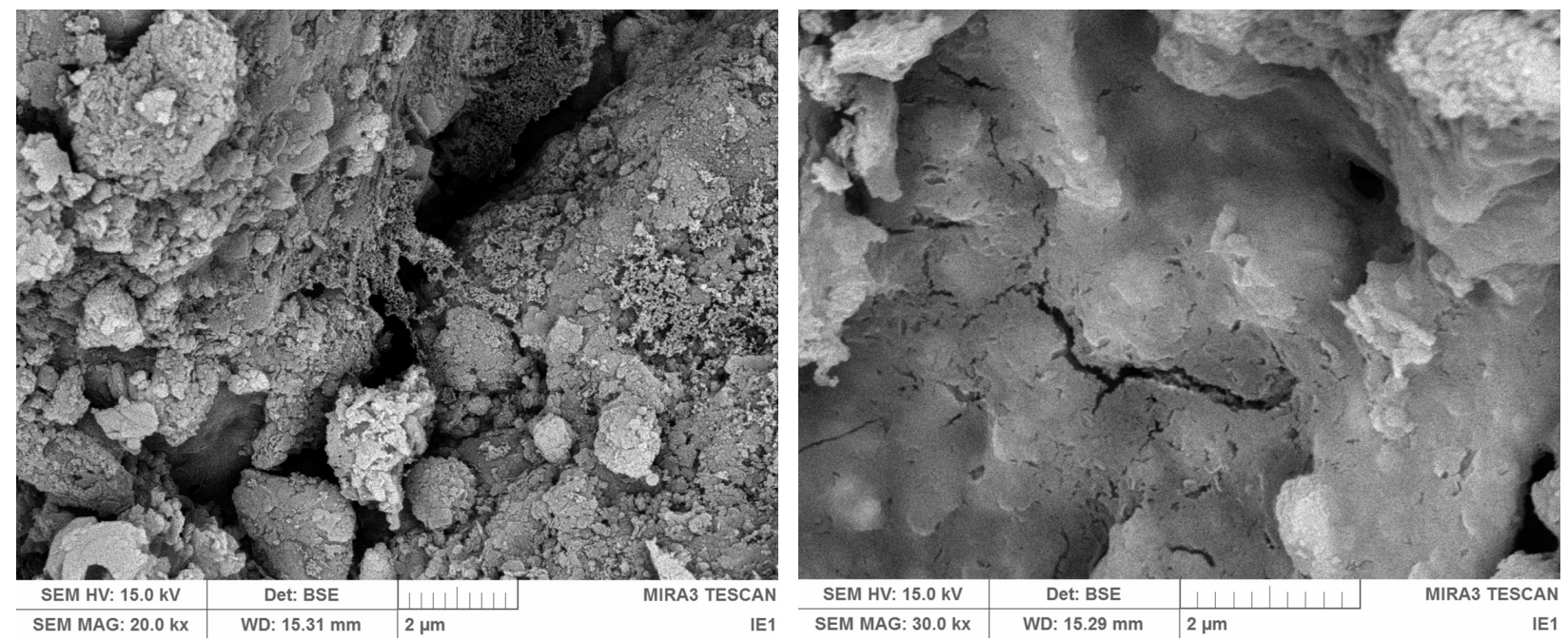

FiguRE 4. SEM/BEI (scanning electron microscope - backscattered electron image). On the left - microstructure of the new binder in the substrate pores as a result of gradual application of lime nano-suspension and alkoxysilane and, on the right, the microstructure of the new binder formed from the lime nano-suspension and alkoxysilane mixture.

fects during stability tests of the mixtures and during consolidation tests on the test panels seemed to be at least adequate as a substitute to purer $99.8 \%$ ethanol. Thus the original supposition that denaturing reagents, above all those featuring a higher content of water, can lower the stability of a suspension was disproven. This finding is especially important from an economic point of view as the price of the $99.8 \%$ ethanol is 10 times higher than of the denatured ethanol.

A further phenomenon, which was investigated during the tests, was the restriction of the white haze forming during or after the application. Apart from the influence of the concentration of the consolidant, something that is dealt with later, there are several basic rules, which it is sensible to adhere to when applying a suspension. The first is the application method. A sprayer with a gas cartridge producing a fine aerosol was found to be the ideal application method for the test panels. This not only ensures a uniform application, but also restricts the amount of fluid applied within a certain period of time. The application is thus easier to regulate than, for example, using a mechanical sprayer. However, in the case of an application onto badly deteriorated materials in situ, the use of a syringe was found to be more suitable as the application could be focused more directly on uneven absorbent substrates.

Another factor capable of influencing the forming of the white haze is the absorbability of the material being strengthened. Where the absorption rate of the surface is low, there is a greater risk of the white haze forming. Conversely, there where the absorption rate is higher, the material being strengthened is capable of absorbing more of the consolidant. This is of course related to the rate of the deterioration of the material - the greater the deterioration, the more intensive the consolidation must be. This, in turn, affects another factor, which is the amount of suspension applied in one cycle. Usually, the ideal method proved to be the application of the material up to the initial saturation of the substrate. If this rule is not observed, the formation of the white haze can be expected with a greater probability.

The final factor is the influence of humidity. It was found that both the relative humidity and subsequent wetting with water restrict the formation of the white haze. Regarding the relative humidity, values of around $60 \%$ are satisfactory. Conversely, a low relative humidity values of around $30 \%$ increase the risk of the white haze formation. Subsequent wetting with spraying of water significantly reduces the formation of the white haze in general. An interesting fact is that even if white haze has formed, it is possible to reduce it, not however eliminate it, by wetting. This finding could be beneficial in cases where the first traces of the white haze appear. Subsequent wetting by spraying could, even after few hours, entirely eliminate these traces. As the experiment showed, wetting after each application cycle of the suspension has a negative effect on the resulting strengthening of the substrate. Therefore, wetting as little as possible is clearly appropriate, e.g., once every $2-3$ cycles, according naturally, to the characteristics of the strengthened material.

Suitable concentration of the suspension and the number of application cycles are of crucial importance. According to the tests undertaken on the panels as well as on genuine historical monuments, the assumption is that in consolidation paint layers 3-6 cycles of a concentration of $5-10 \mathrm{~g}$ of $\mathrm{Ca}(\mathrm{OH})_{2}$ per 1 litre of solvent are suitable. The concentration and number of cycles naturally depend on the type of the material being consolidated. However, experience has shown that more than 6 cycles of a concentration $5 \mathrm{~g}$ of $\mathrm{Ca}(\mathrm{OH})_{2}$ per 1 litre of solvent greatly increase the risk of the white haze formation. 
When comparing individual suspensions with each other, no crucial differences favouring one or the other were noted. For example, pure lime suspensions were used during the consolidation of the medieval wall paintings in St. Vitus Church in the submerged village of Zahrádka near Ledeč nad Sázavou. Here, 4 cycles of a $5 \mathrm{~g} / \mathrm{l}$ concentration were applied to the paint layer.

The application of pure lime suspensions on extremely deteriorated materials was found to be unsuitable due to their relatively low consolidation effect and the risk of the white haze forming as a result of the higher number of application cycles. Therefore, tests were carried out with their mixtures with silicic acid esters and with separate subsequent applications of both consolidants mentioned. Regarding the separate subsequent applications, the test panels displayed a relatively satisfying increase in the paint layer cohesion, but also visual changes in the form of darkening and the white haze creation. Better results as regarding visual impacts and the consolidation were achieved with mixtures. The best mixtures were found to be those in the volume proportions of $1: 1: 1$ (alkoxysilane KSE 100 to alkoxysilane KSE 300 or $300 \mathrm{HV}$ to lime suspension $10 \mathrm{~g} / \mathrm{l})$. Even mixtures in volume proportions of $1: 1: 2$, achieved positively evaluated results. Faring only slightly worse were the $0: 1: 1$ mixtures. When comparing the influence of the unmodified alkoxysilanes (KSE 300) and the specially modified product for improved adhesion to carbonate materials $(K S E 300 H V)$, the unmodified product (KSE 300) performed better.

Two cycles of application of the abovementioned mixtures were not recommended within the framework of the experiment for use on the specific substrate of the test panels. However, they were subsequently used on the severely deteriorated paintings on the ceiling of the chapel of St. Isidor in Křenov. In this case, a combination of consolidandts was selected, in which an alkoxysilane agent in a "concentration" of $100 \mathrm{~g} / \mathrm{l}$ (KSE 100), a mixture of lime suspension concentrated to $10 \mathrm{~g} / \mathrm{l}$ (diluted ZFB 703i) and alkoxysilane guaranteeing the exclusion of gel at a rate of $300 \mathrm{~g} / \mathrm{l}$ (KSE $300 \mathrm{HV}$ ), and finally, ca. 6 cycles of lime suspension concentrated to $5 \mathrm{~g} / \mathrm{l}$ (diluted CaLoSil E25) were successively applied. In the areas where even this combination could not achieve adequate strengthening, the mixture previously described was applied again. The consolidation effect was evaluated positively with subjective methods as regards both the effect of the consolidation and its visual aspect, where no changes were registered.

On the basis of studying the microstructure, as described above, it seems that mixtures of both of these inorganic consolidants could be the appropriate solution to consolidating extremely deteriorated substrates where the use of alkoxysilanes alone may not always be successful. In this regard, their use in the structural strengthening of lime plasters would be interesting.

\section{Conclusion}

Overall, it could be said that lime nano-suspensions are not only appropriate to use for lime based paintings and washes on historical plasters with a light to medium deterioration but, thanks to their material compatibility with the original, they are highly suitable regarding a long-term stability. However, based on the experience with other materials and technologies in the past, it is necessary to note that some unpredictable negative effects of the consolidation can appear after several years, or even decades. Nevertheless, thanks to the high compatibility of this material with the substrate to be strengthened, the risk itself can be considered to be relatively low. This study also gives very detailed instructions for the application of lime nano-suspensions, which enables a good consolidation effect without visual changes to the original material (creation of white haze).

The use of lime nano-suspensions alone for heavily deteriorated materials could be, in some cases, unsatisfactory. It was found that it is possible to achieve the consolidation of such materials by successive applications and mixtures of nano-lime suspensions with alkoxysilanes. From a theoretical point of view, regarding compatibility, the exclusive use of lime consolidants on paint layers is, of course, more suitable. Another reason for precautions when using alkoxysilanes is that after the consolidation, the paint layer could become harder, less elastic and more fragile thus increasing the risk of being damaged in the future. Therefore, the author of this study is of the opinion that the application of alkoxysilanes or their mixtures with lime suspensions is suitable only in those cases where strengthening is not effective with lime suspensions alone. In cases where more effective consolidation is necessary, it is suitable to apply those consolidants mentioned only in as small an amount as possible and to finalize the consolidation with the help of lime suspensions.

\section{REFERENCES}

[1] Ambrosi, M., Dei, L., Giorgi, R., Neto, C., Baglioni, P.: Colloidal Particles of $\mathrm{Ca}(\mathrm{OH})_{2}$ : Properties and Applications to Restoration of Frescoes, In: Langmuir 17 (14), 2001, p. 4251-4255. DOI: 10.1021/la010269b

[2] Ziegenbalg, G., Piaszczynski, E.: The combined application of calcium hydroxide nano-sols and silicic acid ester - a promising way to consolidate stone and mortar. In: 12th International Congress on the Deterioration and Conservation of Stone, New York, 2012, p. 1-8

[3] Jarvie, H., Dobson, P. et al.: Nanoparticle. Encyclopædia Britannica, Available: https://www.britannica.com/science/nanoparticle [2017-02-26]

[4] Toolbox of compatible materials for the conservation of stone, mortar and plaster. IBZ-Salzchemie $\mathrm{GmbH} \&$ Co. KG., Available: http:

//www.ibz-freiberg.de/en/products\#headingOne [2017-02-26] 
[5] Nanorestore Plus®, Technical Information. Available: http://www.csgi.unifi.it/products/plus.html [2017-02-26]

[6] CaLoSiL®Colloidal nano-particles of lime for stone and plaster consolidation, Technical Leaflet. Available: http://www.ibz-freiberg.de/downloads/pdf/ produkte/tm/eng/CaLoSiL_E_IP_NP.pdf [2017-02-26]

[7] Daehne, A., Herm, Ch.: Calcium hydroxide nanosols for the consolidation of porous building materials results from EU-STONECORE. In: Heritage Science Journal 2013, p. 2-4. DOI: 10.1186/2050-7445-1-11

[8] Ibidem, p. 2-4

[9] Stone Conservation for the Refurbishment of Buildings, 7th Framework Programme project (2008-2011)

[10] NANOFORART, Project Abstract. Available: http://www.nanoforart.eu/ [2017-02-26]

[11] Kociánová, I.: Restaurování centrálního výjevu na klenbě kaple sv. Isidora v Křenově. Litomyšl, 2013. Diploma work. University of Pardubice, Faculty of Restoration

[12] Produktprogramm, Baudenkmalpflege, Natursteinkonservierung. Available: http: //www.remmers.de/3852+M5b51a7d4844.0.html\#bot [2017-02-26]

[13] Mora, P., Mora, L., Philippot, P.: Conservation of Wall Paintings, ICCROM, London, 1984. p. 326

[14] Ibidem, p. 326-327

[15] Kociánová, I.: Restaurování centrálního výjevu na klenbě kaple sv. Isidora v Křenově. Litomyšl, 2013. Diploma work. University of Pardubice, Faculty of Restoration
[16] Laurenzi Tabasso M., Simon S.: Testing methods and criteria for the selection/evaluation of products for the conservation of porous building materials, In: Studies in Conservation, 51:sup1, p. 67-82. DOI:

10.1179/sic.2006.51.Supplement-1.67

[17] Henry A. ed.: Stone Conservation: Principles and Practice; Shaftesbury: Donhead, 2006.

[18] More precisely CIE $1976\left(\mathrm{~L}^{*} \mathrm{a} * \mathrm{~b}^{*}\right)$

[19] Drdácký, M. et al.: Innovated water uptake measurements on historic stone surfaces. In: 12th International Congress on the Deterioration and Conservation of Stone, Session V, New York 2012, p. $12-21$

[20] Vandevoorde, D. et al.: Contact sponge method: Performance of a promising tool for measuring the initial water absorption, In: Journal of Cultural Heritage 10 (2009), p. 41-47. DOI: 10.1016/j.culher.2008.10.00

[21] CaLoSiL®Colloidal nano-particles of lime for stone and plaster consolidation, Technical Leaflet. Available: http://www.ibz-freiberg.de/downloads/pdf/ produkte/tm/eng/CaLoSiL_E_IP_NP.pdf [2017-02-26]

[22] Kociánová, I.: Restaurování centrálního výjevu na klenbě kaple sv. Isidora v Křenově. Litomyšl, 2013. Diploma work. University of Pardubice, Faculty of Restoration, p. 55-58, 78-81

[23] Denatured ethanol, Specification. Severochema, Available: http://www.severochema.cz/files/ bezpecnostni-listy/Lih_technicky .pdf [2017-02-26]

[24] Ethanol absolute, Specification. PENTA s.r.o., Available: http://www.pentachemicals.eu/ specifikace/specifikace_193.pdf [2017-02-26] 